\title{
ORDER TREES AND LAMINATIONS OF THE PLANE
}

\author{
David Gabai and William H. Kazez
}

\section{$\S 1$ Preliminary definitions}

A lamination $\lambda$ of $\mathbb{R}^{2}$ is a disjoint union of 1 -manifolds, called leaves, whose union is a closed subset of $\mathbb{R}^{2}$ such that the following properties hold. $\mathbb{R}^{2}$ is covered by charts of the form $\mathbb{R} \times \mathbb{R}$ such that the leaves pass through a given chart in slices of the form $\mathbb{R} \times p t$. The overlap maps are continuous and also smooth when restricted to leaves. Finally, no leaves of $\lambda$ are isolated, that is, leaves of $\lambda$ pass through every chart an infinite number of times. For convenience, figures in this paper are drawn as though they have isolated leaves. Such leaves in the figures are meant to indicate thin bands of parallel leaves.

A lamination $\lambda$ of $\mathbb{R}^{2}$ is essential if no leaves are circles and if about each point of $\mathbb{R}^{2}$ there exists a chart whose intersection with every leaf of $\lambda$ consists of at most one component. These conditions are direct analogues of the "leaves inject on $\pi_{1}$," and "no monogons" conditions that arise in the study of laminations of compact manifolds. Figure 1.1 shows an example of a lamination that is not essential. It is easy to show that leaves of an essential lamination are properly embedded.

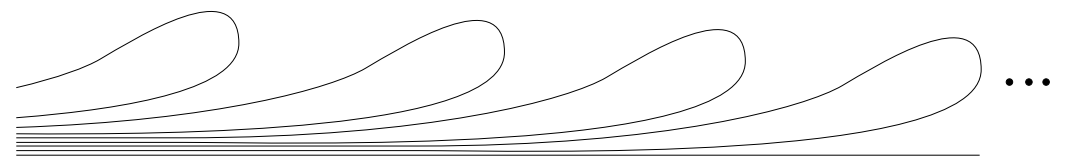

Figure 1.1

Following [GO, Definition 6.9] an order tree is a set $T$ whose elements are called vertices together with a collection $S$ of linearly ordered subsets called segments. Each segment $\sigma$ has distinct least and greatest elements that are denoted $\sigma^{-}$and $\sigma^{+}$respectively. If $\sigma$ is a segment then the inverse of $\sigma$, denoted $-\sigma$, is the same subset with the opposite order. The following axioms must be satisfied:

1) $\sigma \in S \Leftrightarrow-\sigma \in S$

2) If $\tau \subset \sigma \in S$ is a closed subinterval with more than one element then $\tau \in S$.

Received May 1, 1997.

The first authors research was partially supported by NSF Grant DMS-9505253.

We would like to express our thanks to the referee for many useful suggestions. 
3) If $a, b \in T$ then there exists $\sigma_{1}, \ldots, \sigma_{k} \in S$ such that $\sigma_{1}^{-}=a, \sigma_{k}^{+}=b$ and $\sigma_{i}^{+}=\sigma_{i+1}^{-}$for $i=1, \ldots, k-1$.

4) Given a cyclic word $\sigma_{0} \sigma_{1} \ldots \sigma_{k-1}$ (subscripts $\bmod k$ ) with $\sigma_{i}^{+}=\sigma_{i+1}^{-}$ for all $i$, there is a subdivision of the $\sigma_{j}$, that is $\sigma_{j}$ may be written as a concatenation of subsegments, yielding a cyclic word $\rho_{0} \ldots \rho_{n-1}$ which becomes the trivial word when adjacent inverse segments are canceled.

5) If $\sigma_{1}, \sigma_{2} \in S$ and $\sigma_{1} \cap \sigma_{2}=\sigma_{1}^{+}=\sigma_{2}^{-}$then $\sigma_{1} \cup \sigma_{2} \in S$.

An order tree $T$ is an $\mathbb{R}$-order tree if the following two axioms are also satisfied.

6 ) Each $\sigma$ is order isomorphic to a closed interval in $\mathbb{R}$.

7) $T$ is a countable union of segments.

Two order trees are isomorphic if there is a bijection of their vertices that sends segments to segments. Order trees $T$, or more precisely, the vertices of $T$, are topologized by giving segments the order topology and defining $U \subset T$ to be open if and only if $U \cap \sigma$ is open in $\sigma$ for all $\sigma$. For $\mathbb{R}$-order trees, any choice of order isomorphism between segments and a closed interval in $\mathbb{R}$ must be a homeomorphism so, when convenient, segments will be identified with closed intervals in $\mathbb{R}$.

Some examples of order trees are:

(1) Countable trees. The vertices are all points of the tree and the segments are all oriented embedded arcs in the tree.

(2) Non-Hausdorff 1-manifolds. The vertices are all points in the manifold and the segments are all oriented embedded arcs in the tree. In Figure 1.2 the horizontal line approaches both $A$ and $B$. Axiom 4 is much weaker than the statement that there exists a unique segment between any two vertices.

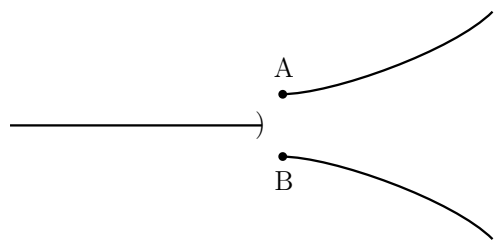

Figure 1.2

(3) $\mathbb{R}$-trees. [MS] show that $\mathbb{R}$-trees may be topologically characterized as follows. A metric space is an $\mathbb{R}$-tree if every two points are joined by a unique arc and if every such arc is isometric to an interval. Thus $\mathbb{R}$-trees are order trees.

Later we shall give examples of $\mathbb{R}$-order trees which are not first countable and in which segments may not be closed. $\mathbb{R}$-order trees are $T_{1}$.

Let $\lambda$ be an essential lamination in $\mathbb{R}^{2}$. An embedded arc $\alpha:[0,1] \rightarrow \mathbb{R}^{2}$ is called efficient (with respect to $\lambda$ ) if it is transverse to the leaves of $\lambda$ and if it intersects each leaf of $\lambda$ in at most one point.

The path metric on a complementary region of a lamination is given by letting the distance between two points be the infimum of the lengths of all paths in that complementary region that connect the two points. A closed complementary 
region of $\lambda$ is the completion, in the path metric, of a complementary region. This is the same as the closure in $\mathbb{R}^{2}$ of the complementary region if $\lambda$ is essential. $\mathrm{A}$ leaf of $\lambda$ is a non-boundary leaf if it is not contained in any closed complementary region.

The order tree $T(\lambda)$ associated to $\lambda$ is defined as follows. The vertices of $T(\lambda)$ are the closed complementary regions of $\lambda$ together with the non-boundary leaves of $\lambda$. Let $\alpha$ be an efficient arc and let $\nu: \mathbb{R}^{2} \rightarrow T(\lambda)$ be the natural map. If $\nu(\alpha)$ contains more than one point then it is defined to be a segment of $T$. $T(\lambda)$ is the natural analogue of the leaf space of a foliation.

Proposition 1.1. If $\lambda$ is an essential lamination of $\mathbb{R}^{2}$ then $T(\lambda)$ is an $\mathbb{R}$-order tree and $\nu: \mathbb{R}^{2} \rightarrow T(\lambda)$ is a quotient map.

Proof. See [GO, Proposition 6.10] for the original version which they established for codimension-1 essential laminations of 3-dimensional manifolds. We make only a few comments on the proof.

The hypothesis that $\lambda$ is essential is used to show that every segment has a linear order and that every vertex of $T$ is contained in some segment of $T$ which is part of what is needed to prove Axiom 3.

Let $\alpha_{0}, \ldots, \alpha_{k-1}$ be efficient arcs corresponding to the segments of the cyclic word $\sigma_{0} \ldots \sigma_{k-1}$. Let $D$ be a disk in $\mathbb{R}^{2}$ that contains $\cup_{i} \alpha_{i}$ and such that $\partial D$ has isolated tangencies with $\lambda$. It is not hard to see by considering families of parallel leaves of $\lambda \cap D$ that the "leaf space" of $\lambda \cap D$ is just a finite tree. Axiom 4 then follows since it is known for trees.

Axiom 6 requires the hypothesis that a lamination contain no isolated leaves. One can mimic the construction of the Cantor function to build a function that is constant on the intersections of an efficient arc with the closed complementary regions of $\lambda$ and thereby establish Axiom 6 .

In Figure 1.3 the associated $\mathbb{R}$-order tree is not Hausdorff and in Figure 1.4 the associated $\mathbb{R}$-order tree is not first countable.

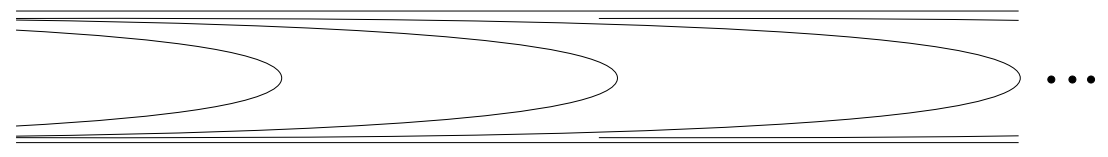

Figure 1.3

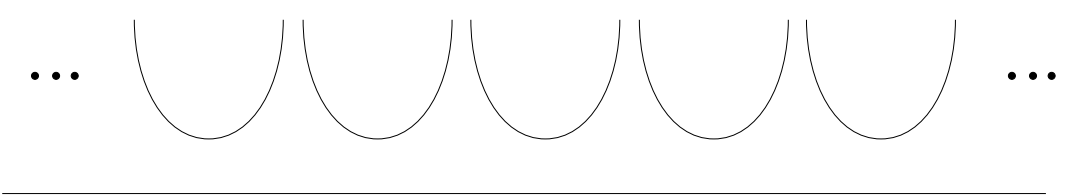

Figure 1.4 


\section{$\S 2$ Cyclic orders}

Let $\ell_{1}, \ldots, \ell_{n}$ be a disjoint collection of properly embedded copies of $\mathbb{R}$ in $\mathbb{R}^{2}$ such that each bounds a half space $H_{i} \subset \mathbb{R}^{2}$ and $H_{i} \cap H_{j}=\emptyset$ for $i \neq j$. The natural cyclic order on $\left\{\ell_{i}\right\}$ is given by picking a point $x \notin \cup_{i=1}^{n} H_{i}$, disjoint arcs $\alpha_{i}$ from $x$ to each $\ell_{i}$, and then ordering $\left\{\ell_{i}\right\}$ by the cyclic order on the $\alpha_{i}$.

A cyclic ordering on an $\mathbb{R}$-order tree is, roughly, a cyclic order on sets of segments with only a vertex in common, and a cyclic order corresponding to segments which differ only by a vertex. More precisely

1) If $\left\{\sigma_{i}\right\}$ is a finite set of segments such that $\sigma_{i}^{-}=\sigma_{j}^{-}$for all $i, j$ and $\left(\sigma_{i}-\sigma_{i}^{-}\right) \cap\left(\sigma_{j}-\sigma_{j}^{-}\right)=\emptyset$ for all $i \neq j$, then there is a cyclic order of $\left\{\sigma_{i}\right\}$. Moreover, this order is induced if $\left\{\sigma_{i}\right\}$ is replaced by a subset of $\left\{\sigma_{i}\right\}$ or by a set of initial subsegments of $\left\{\sigma_{i}\right\}$.

2) If $\left\{\tau_{i}\right\}$ is a finite collection of segments such that $\tau_{i}^{+} \neq \tau_{j}^{+}$for $i \neq j$ and $\tau_{i}-\tau_{i}^{+}=\tau_{j}-\tau_{j}^{+}$for all $i, j$ then there is a cyclic order on the set $\{\tau\} \cup\left\{\tau_{i}^{+}\right\}$where $\tau=\tau_{i}-\tau_{i}^{+}$. Moreover, this order is induced if $\left\{\tau_{i}\right\}$ is replaced by a subset of $\left\{\tau_{i}\right\}$ or a set of terminal subsegments of $\left\{\tau_{i}\right\}$.

See Definition 6.5 for an alternative definition of cyclic ordering.

Proposition 2.1. An essential lamination $\lambda$ of $\mathbb{R}^{2}$ determines a cyclic order on $T(\lambda)$.

Proof. Segments with only a common vertex may be ordered by first picking points in them corresponding to leaves of $\lambda$ and then using the natural ordering of these leaves.

Given segments $\left\{\tau_{i}\right\}$ which differ only by $\tau_{i}^{+}$, for each $i$ pick a leaf in the subset of $\mathbb{R}^{2}$ corresponding to $\tau_{i}^{+}$(which may be just a leaf or an entire closed complementary region) and pick a leaf in $\lambda$ corresponding to a point in $\tau=$ $\tau_{i}-\tau_{i}^{+}$. Order $\{\tau\} \cup\left\{\tau_{i}^{+}\right\}$by using the natural ordering of the corresponding leaves.

It is straightforward to check that this defines a cyclic ordering of $T(\lambda)$.

\section{$\S 3$ Decomposition of $\mathbb{R}$-order trees}

An $\mathbb{R}$-order tree $T$ is, by definition, a countable union of segments $\sigma_{1}, \sigma_{2}, \ldots$. Let $a \in T$. For each $i$ there exists a finite, possibly empty, collection of segments connecting $a$ and $\sigma_{i}^{-}$. After augmenting $\left\{\sigma_{i}\right\}$ by these segments and reindexing we may assume

1) $T=\bigcup_{i=1}^{\infty} \sigma_{i}$

2) $\left(\cup_{i=1}^{n} \sigma_{i}\right) \cap \sigma_{n+1} \neq \emptyset$ for all $n \geq 1$.

Let $T_{n}=\bigcup_{i=1}^{n} \sigma_{i}$. Since $T_{n}$ is connected, Axiom 4 implies that if $\sigma_{n+1}^{+}, \sigma_{n+1}^{-} \in$ $T_{n}$, then $\sigma_{n+1} \subset T_{n}$. Thus after possibly reindexing and reorienting we may assume

3) $T_{n} \cap \sigma_{n+1}$ is an initial segment of $\sigma_{n+1}$, for $n \geq 1$. 
Identify $\sigma_{n+1}$ with an interval $[a, c] \subset \mathbb{R}$. Then there are three possibilities for $T_{n} \cap \sigma_{n+1}$. These are $[a, a],[a, b)$ with $b \leq c$ and $[a, b]$ with $a<b<c$. By subdividing and reindexing we may replace Condition 3 by

$\left.3^{\prime}\right) T_{n} \cap \sigma_{n+1}$ is equal to either $\sigma_{n+1}^{-}$or $\sigma_{n+1}-\sigma_{n+1}^{+}$.

$T_{n}$ may be thought of as a finite "non-Hausdorff tree".

Proposition 3.1. An $\mathbb{R}$-order tree $T$ contains segments $\sigma_{1}, \sigma_{2}, \ldots$ such that if $T_{n}=\cup_{i=1}^{n} \sigma_{i}$ then $T=\cup_{n=1}^{\infty} T_{n}$ and for each $n \geq 1$ either

a) $T_{n-1} \cap \sigma_{n}=\sigma_{n}^{-}$

b) $T_{n}-T_{n-1}=\sigma_{n}^{+}$

\section{$\S 4$ Existence of laminations}

The next lemma is a local existence result for laminations that will be used to create complementary regions as needed.

Lemma 4.1. Let $\sigma$ be a segment of an $\mathbb{R}$-order tree and let $C=\left\{c_{1}, c_{2}, \ldots\right\}$ be a countable subset of $\sigma$ that does not contain the endpoints of $\sigma$. There exists a function $f: I \rightarrow \sigma$ such that $f(0)=\sigma^{-}, f(1)=\sigma^{+}, f^{-1}(x)$ consists of one point if $x \notin C$, and $f^{-1}(x)$ is a closed interval if $x \in C$.

The points of $\overline{f^{-1}(C)}$ may be thought of as a codimension-1 lamination $\lambda^{0}$ of $I$ in which case $f$ induces an isomorphism $T\left(\lambda^{0}\right) \rightarrow \sigma$.

Proof. Let $U$ be the complement of the standard Cantor "middle third" subset of $I$. Inductively pick components $U_{i}$ of $U$ so that $c_{i}<c_{j}$ in $\sigma$ if and only if $x_{i}<x_{j}$ for all $x_{i} \in U_{i}, x_{j} \in U_{j}$. Let $\lambda^{0} \equiv I-\cup_{i=1}^{\infty} U_{i}$.

Next by mimicking the construction of the Cantor function there is a semimonotone continuous function $f: I \rightarrow \sigma$ such that $f(0)=\sigma^{-}, f(1)=\sigma^{+}$, $f\left(U_{i}\right)=c_{i}$ and $f$ is constant on no interval not in $\cup_{i=1}^{\infty} U_{i}$.

The idea behind the next theorem is very simple. Suppose that a lamination $\lambda_{n}$ has been built such that $T\left(\lambda_{n}\right) \cong T_{n}$ and suppose there is a vertical gap, $[a, b] \times[n, \infty)$ in the lamination. That is $(a, b) \times[n, \infty) \subset \mathbb{R}^{2}-\lambda_{n}$ and $\{a, b\} \times$ $[n, \infty) \subset \lambda_{n}$. Figure 4.1 shows a bit of lamination that can be inserted into the gap, and Figure 4.1 shows how $\lambda_{n}$ can be perturbed near the gap. The effect on $T_{n}$ is just as in Proposition $3.1 \mathrm{a}$ ) and b). Much of the proof of Theorem 4.2 consists of anticipating where the vertical gaps will be needed.

Theorem 4.2. Every cyclically ordered $\mathbb{R}$-order tree $T$ is isomorphic to the associated tree of some essential lamination $\lambda$ of $\mathbb{R}^{2}$.

Proof. Let $T=\cup_{n=1}^{\infty} T_{n}$ and $T_{n}=\cup_{i=1}^{n} \sigma_{i}$ be a decomposition of $T$ satisfying the conclusion of Proposition 3.1.

In the construction of $\lambda$, complementary regions must be constructed in different ways depending whether $\sigma_{n}$ satisfies property a) or property b) of Proposition 3.1. Let $A=\left\{n \mid \sigma_{n}\right.$ satisfies property a) $\}$ and let $B=\left\{n \mid \sigma_{n}\right.$ satisfies 
property b)\}. For $n \in B, \sigma_{n}^{+}$is a "non-Hausdorff point of $T_{n}$ " and we let $N_{n}$ be the points in $T_{n}$ that $\sigma_{n}^{+}$can not be separated from . That is, $N_{n}=\left\{\tau^{+} \mid \tau\right.$ is a segment in $T_{n-1}$ such that there exists a segment $\sigma \subset T_{n}$ with $\sigma^{+}=\sigma_{n}^{+}$and $\left.\tau-\tau^{+}=\sigma-\sigma^{+}\right\}$. The points for which complementary regions will be needed are $C=\left(\cup_{n \in A}\left\{\sigma_{n}^{-}\right\}\right) \cup\left(\cup_{n \in B} N_{n}\right)$.

For each $n \in A$, that is $n$ for which a new edge is added to $T_{n-1}$, let $C_{n}=$ $\left(\sigma_{n} \cap C\right)-\left(\left\{\sigma_{n}^{-} \cup \sigma_{n}^{+}\right\}\right)$. By Lemma 4.1 there corresponds to $C_{n} \subset \sigma_{n}$ a closed set $\lambda_{n}^{0}$ of $I$. The product lamination corresponding to $\sigma_{n}$ is defined to be $\lambda_{n}^{0} \times \mathbb{R} \subset$ $[0,1] \times \mathbb{R}$. Ultimately product laminations will be embedded in $\mathbb{R}^{2}$ as shown in Figure 4.1.

Given a boundary leaf of a lamination we may introduce a prong as follows. Choose coordinates such that $(0,0)$ is a point on a boundary leaf $0 \times(-1,1)$ of a vertical product lamination of $[0,1] \times(-1,1)$. The only change in the leaves of the lamination is to remove the point $(0,0)$, but since the goal is to create a lamination of $\mathbb{R}^{2}$ these new leaves are embedded as shown in Figure 4.2.

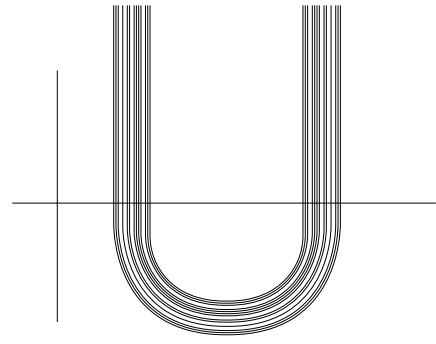

Figure 4.1

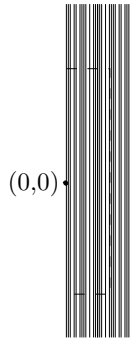

The portion of the prong above the horizontal dotted line is called a vertical prong.

$\lambda$ will be built from essential laminations $\lambda_{n}$ which are constructed inductively with the following properties:

1) For $n+1 \in A, \lambda_{n+1}$ is the disjoint union of $\lambda_{n}$ and a product lamination corresponding to $\sigma_{n+1}$

2) For $n+1 \in B, \lambda_{n+1}$ is obtained by adding a prong to $\lambda_{n}$ as in Figure 4.2

3) $T\left(\lambda_{n+1}\right)$ is isomorphic to $T_{n+1}$ where the cyclic order on $T_{n+1}$ is induced from $T$.

4) There exists $y_{n+1}$ such that $\lambda_{n+1}-H_{y_{n+1}}$ is a union of vertical prongs, where $H_{\ell}$ denotes the halfspace $\left\{(x, y) \in \mathbb{R}^{2} \mid y \leq \ell\right\}$.

The complementary regions required in 1) and 2) are guaranteed by the choice of product laminations corresponding to the $\sigma_{n}^{\prime} s$. The details of the construction follow.

$\lambda_{1}$ is the product lamination corresponding to $\sigma_{1}, y_{1}=0$, and $\lambda_{1}-H_{y_{1}}$ is a union of vertical lines. See Figure 4.1.

$\lambda_{n+1}$ is obtained from $\lambda_{n}$ in one of two ways. If $n+1 \in A$ let $\left\{\rho_{i}\right\}$ be a set, of maximum cardinality, of segments of $T_{n}$ such that $\rho_{i}^{+}=\sigma_{n+1}^{-}$and 
$\left(\rho_{i}-\rho_{i}^{+}\right) \cap\left(\rho_{i}-\rho_{j}^{+}\right)=\emptyset$ for $i \neq j$. Let $\ell_{i}$ be the leaf of $\lambda_{n}$ corresponding to $\rho_{i}^{+}$, that is, it is the leaf in the boundary of the region corresponding to $\sigma_{n+1}^{-}$that is limited on by leaves in $\rho_{i}-\rho_{i}^{+}$.

Choose $y_{n+1}>y_{n}$ such that $\mathbb{R} \times\left\{y_{n+1}\right\}$ intersects each $\ell_{i}$ in two points. By construction the cyclic order on the $\ell_{i}$ given by intersecting with $\mathbb{R} \times\left\{y_{n+1}\right\}$ (and identifying $-\infty$ with $+\infty$ ) agrees with the cyclic order on $T_{n}$. Say that in the cyclic order on $\left\{\rho_{i}\right\} \cup\left\{\sigma_{n+1}\right\}$ in $T_{n+1}, \sigma_{n+1}$ falls between $\rho_{1}$ and $\rho_{2}$ (possibly with $\left.\rho_{1}=\rho_{2}\right)$.

Let $(a, b)$ be the shortest interval in $\mathbb{R} \times\left\{y_{n+1}\right\}$ connecting $\ell_{1}$ and $\ell_{2}$. It follows that $(a, b) \subset \mathbb{R}^{2}-\lambda_{n} \cdot \lambda_{n+1}$ is defined to be the disjoint union of $\lambda_{n}$ and the product lamination corresponding to $\sigma_{n+1}$ that is embedded in such a way that its intersection with $\mathbb{R} \times\left\{y_{n+1}\right\}$ is contained in $(a, b)$ and above $\mathbb{R} \times\left\{y_{n+1}\right\}$ it is a union of vertical lines.

If $n+1 \in B$ then $T_{n+1}=T_{n} \cup \sigma_{n+1}^{+}$. Choose a segment $\tau$ of $T_{n+1}$ with $\tau^{+}=\sigma_{n+1}^{+}$and choose segments $\tau_{i}$ of $T_{n}$ such that any two elements of the set $\left\{\tau_{i}\right\} \cup\{\tau\}$ differ only by their terminal endpoint and such that $\left\{\tau_{i}\right\} \cup\{\tau\}$ has maximum cardinality among all such sets of segments.

By construction, for each $\tau_{i}^{+}$there is a corresponding closed complementary region of $\lambda_{n}$. Let $\ell_{i}$ be the boundary leaf limited on by leaves corresponding to $\tau_{i}-\tau_{i}^{+}$. Choose $y_{n+1}>y_{n}$ such that $\ell_{i}$ intersects $\mathbb{R} \times\left\{y_{n+1}\right\}$ in two points. By construction the natural cyclic ordering on $\left\{\ell_{i}\right\}$ corresponds to the cyclic order on $T_{n}$. In the cyclic order on $\left\{\tau_{i}^{+}\right\} \cup\left\{\tau^{+}\right\} \cup\{\tau\}$ given by $T_{n+1}$ say $\tau^{+}$is adjacent to $\tau_{1}^{+}$and $\tau_{2}^{+}$(and possibly $\tau_{1}^{+}=\tau_{2}^{+}$). Let $b \in \ell_{1} \cap\left(\mathbb{R} \times y_{n+1}\right)$ be the closest element to $\ell_{2} \cap\left(\mathbb{R} \times y_{n+1}\right)$ (either element will do if $\left.\tau_{1}^{+}=\tau_{1}^{+}\right)$.

Then there exists an interval $(a, c) \subset \mathbb{R} \times\left\{y_{n+1}\right\}$ containing $b$ such that one component of $(a, c)-b$ is contained in the complement of $\lambda_{n}$ and the other component corresponds to a subset of $\tau_{1} \cdot \lambda_{n+1}$ is obtained by adding a prong to $\lambda_{n}$ near $(a, c)$. By slightly increasing $y_{n+1}$ the portion of the prong above $y_{n+1}$ may be chosen to be vertical.

The desired lamination $\lambda$ is defined by letting $\lambda \cap\left(H_{y_{n+1}}-H_{y_{n}}\right)=\lambda_{n+1} \cap$ $\left(H_{y_{n+1}}-H_{y_{n}}\right)$.

\section{$\S 5$ Basic constructions}

We state without proof a few basic constructions used in studying laminations. The next proposition is called splitting along leaves and is used to replace nonboundary leaves by closed complementary regions.

Proposition 5.1. Let $\ell_{1}, \ell_{2}, \ldots$ be non-boundary leaves of an essential lamination $\lambda$. There exists a lamination $\lambda^{\prime}$ with closed complementary regions $N_{1}, N_{2}, \ldots$ such that $N_{i} \cong \ell_{i} \times I$. Furthermore if $\sim$ denotes the equivalence relation $(x, s) \sim(x, t)$ for $x \in \cup_{i} \ell_{i}$ and $s, t \in I$ then $\left(\mathbb{R}^{2} / \sim, \lambda^{\prime} / \sim\right) \cong\left(\mathbb{R}^{2}, \lambda\right)$. 
Proposition 5.2. If $\lambda^{\prime}$ is obtained by splitting along leaves of $\lambda$ then $T\left(\lambda^{\prime}\right) \cong$ $T(\lambda)$.

Definition. A fibred neighborhood of a lamination $\lambda$ is a disjoint union $N(\lambda) \subset$ $\mathbb{R}^{2}$ of closed intervals transverse to the leaves of $\lambda$ such that

1) $\lambda \subset N(\lambda)$.

2) The endpoints of each interval in the disjoint union are contained in $\lambda$.

3) For each point in $N(\lambda)$ there is an open set $U \subset \mathbb{R}^{2}$ such that $U \cap$ $N(\lambda)$ is a union of $I$-bundle fibres and $U \cap N(\lambda)$ is homeomorphic, by a homeomorphism which sends fibres to vertical lines, to one of the subsets of $\mathbb{R}^{2}$ shown in Figure 5.1.
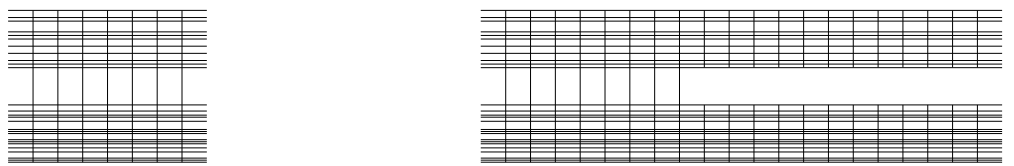

Figure 5.1

Proposition 5.3. Every lamination $\lambda \subset \mathbb{R}^{2}$ may be split along leaves to produce a lamination $\lambda^{\prime}$ which has a fibred neighborhood $N\left(\lambda^{\prime}\right)$. If $\lambda$ is essential, $N\left(\lambda^{\prime}\right)$ may be chosen so that each leaf of $\lambda^{\prime}$ intersects each I-bundle fibre of $N\left(\lambda^{\prime}\right)$ at most once.

The next lemma can be used to compare different $I$-bundle fibres in a fibred neighborhood.

Lemma 5.4. Let $\alpha$ and $\beta$ be efficient arcs with disjoint interiors that connect leaves $\ell_{0}$ and $\ell_{1}$ of an essential lamination of $\mathbb{R}^{2}$. Then $\alpha \cup \beta$ together with arcs joining $\partial \alpha$ and $\partial \beta$ in $\ell_{0}$ and $\ell_{1}$ bound a disk $F$, and $\lambda \mid F$ is a product lamination.

Proof. This follows from the Jordan Curve Theorem. For codimension-1 laminations of 3-manifolds the same lemma holds [GO, Lemma 6.5]. In that setting the lemma is equally useful, but the proof is more subtle.

Definition. A product complementary region is a complementary region with two boundary components.

With a little care it is possible to define an inverse operation called collapsing in which product complementary regions are replaced by single leaves. This operation will not be used, but it may be useful to use this in other situations.

\section{$\S 6$ Equivalence of laminations}

Let $f_{*}: T(\lambda) \rightarrow T\left(\lambda^{\prime}\right)$ be an isomorphism of cyclically ordered $\mathbb{R}$-order trees, that is an isomorphism of order trees that preserves the cyclic order on the trees. Our goal is to show that $f_{*}$ is induced by a homeomorphism $f$. We shall do this locally first, that is for individual closed complementary regions and neighborhoods of efficient arcs, and then introduce an exhaustion technique for extending these local results. 
Let $C$ be a closed complementary region of $\lambda$ and let $\left\{\ell_{i}\right\}$ be the components of $\partial C$ with the natural order. Choose segments $\sigma_{i} \subset T(\lambda)$ with $\sigma_{i}^{-}=[C]$, where $[C] \in T(\lambda)$ is the point corresponding to $C,\left(\sigma_{i}-\sigma_{i}^{-}\right) \cap\left(\sigma_{j}-\sigma_{j}^{-}\right)=\emptyset$ for $i \neq j$, and $\sigma_{i}-\sigma_{i}^{-}$limits on $\ell_{i}$. Then $\left\{f_{*}\left(\sigma_{i}\right)\right\}$ are a collection of segments in $T\left(\lambda^{\prime}\right)$ with the same intersection and order properties as $\left\{\sigma_{i}\right\}$. If $\left\{\sigma_{i}\right\}$ has cardinality not equal to 2 , then $f_{*}([C])$ corresponds to a complementary region of $T\left(\lambda^{\prime}\right)$ which will be denoted $f(C)$ and there exists a homeomorphism $f: C \stackrel{\cong}{\longrightarrow} f(C)$ which preserves the cyclic ordering.

If $C$ has exactly 2 boundary components then $C$ is a product region and it may be that there is a non-boundary leaf $\ell^{\prime}$ of $\lambda^{\prime}$ such that $f_{*}[C]=\left[\ell^{\prime}\right]$. By Lemma 4.1 and Proposition 5.1 we have

Proposition 6.1. Let $\lambda$ and $\lambda^{\prime}$ be essential laminations of $\mathbb{R}^{2}$ and let $f_{*}$ : $T(\lambda) \rightarrow T\left(\lambda^{\prime}\right)$ be an isomorphism. There exist leaves of $\lambda$ and $\lambda^{\prime}$ such that after splitting, and still denoting the new laminations by $\lambda$ and $\lambda^{\prime}, f_{*}$ induces a bijection between points corresponding to closed complementary regions and such complementary regions are homeomorphic.

Definition 6.2. A product neighborhood $P$ of an efficient arc $\alpha$ for a lamination $\lambda$ is an embedding of $[-1,1] \times I$ such that $0 \times I$ maps to $I$, vertical lines are mapped to efficient arcs and the preimage of $\lambda$ is a union of horizontal intervals. If the endpoints of $\alpha$ are in closed complementary regions $C_{1}$ and $C_{2}$ we say $P$ connects $C_{1}$ and $C_{2}$.

In the next proposition we assume $\lambda$ and $\lambda^{\prime}$ have been split along leaves as required to satisfy Proposition 6.1.

Proposition 6.3. Let $f_{*}: T(\lambda) \rightarrow T\left(\lambda^{\prime}\right)$ be an isomorphism and let $\sigma \subset T(\lambda)$ be a segment. Then there exist product neighborhoods of efficient arcs $\alpha, \beta$, for $\lambda$ and $\lambda^{\prime}$ respectively, which are homeomorphic by a homeomorphism inducing $\left.f_{*}\right|_{\sigma}: \sigma \rightarrow f_{*}(\sigma)$.

Proof. By definition there are efficient arcs $\alpha$ and $\beta$ corresponding to $\sigma$ and $f_{*}(\sigma)$. Since $f_{*}$ preserves points corresponding to complementary regions, $\left.f_{*}\right|_{\sigma}$ induces a homeomorphism from $\alpha \cap \lambda$ to $\beta \cap \lambda^{\prime}$. This extends linearly over complementary regions to a homeomorphism from $\alpha$ to $\beta$ which then extends to the required homeomorphism of product neighborhoods.

The following very useful exhaustion theorem was originally proved in [GO, Construction 6.11] for 3-dimensional manifolds and in the form stated it can be found in [GK, Lemma 4.1]. The proof requires that the lamination $\lambda$ be contained in a fibred neighborhood which can always be arranged by Proposition 5.3.

Theorem 6.4. There is an exhaustion $X_{1} \subset X_{2} \subset \ldots$ of $\mathbb{R}^{2}$ such that each $X_{i}$ is a finite union of disjoint closed complementary regions of $\lambda$ that are connected by a finite disjoint collection of product neighborhoods of efficient arcs. Moreover, $X_{1}$ is a single closed complementary region of $\lambda$ and $X_{n+1}$ is a union of $X_{n}$ and 
a product neighborhood of an efficient arc $P$ and/or a closed complementary region $C$. The configuration of $X_{n}, P$ and/or $C$ is given by one of the following possibilities:

1) $P$ is a thickening of a product neighborhood $Q$ of an efficient arc in $X_{n}$. That is, $P$ contains $Q$, connects the same complementary regions as $Q$ and is disjoint from all other product neighborhoods of efficient arcs in the decomposition of $X_{n}$.

2) $X_{n+1}=X_{n} \cup P \cup C$ where $X_{n} \cap C=\emptyset$ and $P$ is a product neighborhood connecting a closed complementary region of $X_{n}$ and $C . P$ does not intersect any other product neighborhood in the decomposition of $X_{n}$.

3) $X_{n+1}=X_{n} \cup P \cup C$ where $C \cap X$ is contained in product neighborhoods $Q_{1}, \ldots, Q_{k}$ and each $Q_{i}$ connects a $C_{0} \subset X_{n}$ to some $C_{i} \subset X_{n} . P$ is a product neighborhood connecting $C_{0}$ and $C$ that contains the portions of $Q_{1}, \ldots, Q_{k}$ between $C_{0}$ and $C$. In the decomposition of $X_{n+1}$, each $Q_{i}$ should be replaced by the portion of it that lies between $C$ and $C_{i}$.

It is useful to think of each $X_{n}$ as a tree with vertices $C_{i}$ and edges $P_{j}$. See Figure 6.1.

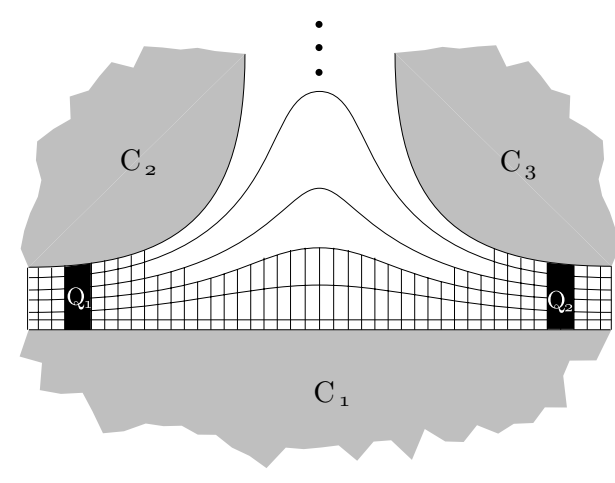

$\mathrm{X}_{3}$

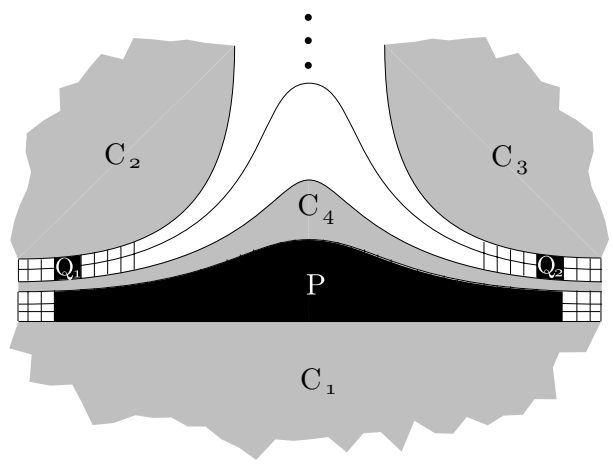

$\mathrm{X}_{4}$

Figure 6.1

Let $P_{1}, \ldots, P_{k}$ be edges of $X_{n}$ such that $P_{i}$ connects $C_{0}$ to $C_{i}$. When $X_{n}$ is thought of as a tree embedded in $\mathbb{R}^{2}$ there is an induced cyclic order on $\left\{P_{i}\right\}$. This is of course related to the cyclic order on $\lambda$. To see this relation we define a stronger form of cyclic ordering and then point out that it is in fact equivalent to the original cyclic ordering condition.

Definition 6.5. An $\mathbb{R}$-order tree $\lambda$ is strongly cyclically ordered if there is a cyclic order on each finite set of segments $\sigma_{1}, \ldots, \sigma_{k}$ that satisfy $\sigma_{i}^{-}=\sigma_{j}^{-}$for all $i, j$ and $\sigma_{i}^{+} \notin \sigma_{j}$ for all $i \neq j$. Moreover this ordering is induced if $\left\{\sigma_{i}\right\}$ is replaced by a subset of itself or by a set of initial subsegments satisfying the same conditions.

Proposition 6.6. A cyclic ordering of an $\mathbb{R}$-order tree $T$ can be extended in a unique way to a strong cyclic ordering. 
Proof. By Theorem 4.2, $T$ is realized by an essential lamination of $\mathbb{R}^{2}$. The existence of an extension to a strong cyclic ordering is then given by the natural ordering on the leaves corresponding to $\sigma_{1}^{+}, \ldots, \sigma_{k}^{+}$in $\mathbb{R}^{2}$. For uniqueness it is enough to consider the case that there are just 3 segments $\left\{\sigma_{i}\right\}$. It is then possible to restrict to subsegments and use the properties of a cyclic order to establish uniqueness.

Returning to the ordering on the edges $P_{1}, \ldots, P_{k}$ of $X_{n}$ discussed above, we see that it corresponds to the (strong) cyclic order on $\lambda$.

Proposition 6.7. Given an isomorphism $f_{*}: T(\lambda) \rightarrow T\left(\lambda^{\prime}\right)$ of cyclically ordered $\mathbb{R}$-order trees and assuming $\lambda$ and $\lambda^{\prime}$ have been split along leaves as needed in Proposition 6.1, then there exists an injective map $f:\left(\mathbb{R}^{2}, \lambda\right) \rightarrow\left(\mathbb{R}^{2}, \lambda^{\prime}\right)$ with image containing the closed complementary regions of $\lambda^{\prime}$ that induces $f_{*}$.

Proof. Let $X_{1} \subset X_{2} \subset \ldots$ be an exhaustion of $\mathbb{R}^{2}$ as in Theorem 6.4 for the lamination $\lambda$. Inductively define $f:\left(\mathbb{R}^{2}, \lambda\right) \rightarrow\left(\mathbb{R}^{2}, \lambda^{\prime}\right)$ that induces $f_{*}$ as follows.

Let $f \mid X_{1}$ be a homeomorphism as guaranteed by Proposition 6.1. Assume $f$ has been defined on $X_{n}$ and extend $f$ to $X_{n+1}$ by considering the cases of Theorem 6.4.

1) If $P$ is a thickening of a product neighborhood $Q$, then by construction $f \mid Q$ maps $Q$ homeomorphically to a product neighborhood $f(Q)$. Let $f(P)$ be a thickening of $f(Q)$ consistent with the definition of $f$ on the closed complementary regions connected by $P$, and let $f \mid P$ be an extension of $f \mid Q$ to a homeomorphism from $(P, P \cap \lambda)$ to $\left(f(P), f(P) \cap \lambda^{\prime}\right)$.

2) If $X_{n+1}=X_{n} \cup P_{1} \cup C_{1}$ with $X_{n} \cap C_{1}=\emptyset$. Say $P_{1}$ connects $C_{0} \subset X_{n}$ to $C_{1}$ and let $P_{2}, \ldots, P_{k} \subset X_{n}$ be all edges of $X_{n}$ which connect the leaf $\ell$ of $C_{0}$ containing $P_{1} \cap C_{0}$ and say $P_{i}$ also connects $C_{i} \subset X_{n}$. By induction $f: X_{n} \rightarrow \mathbb{R}^{2}$ is an injective map that preserves the cyclic order induced from $T(\lambda)$ and $T\left(\lambda^{\prime}\right)$.

By Propositions 6.1 and 6.3 we can find an injection $C_{0} \cup P_{1} \cup C_{1} \hookrightarrow \mathbb{R}^{2}$ that realizes the order tree structure. To choose such a map that is also an extension of $f \mid X_{n}$ we must know that the linear ordering on $P_{2}, \ldots, P_{k}$ induced by $\ell$ is preserved. At first glance, $f$ only preserves a cyclic ordering of $P_{2}, \ldots, P_{k}$.

Since $\ell$ is a boundary leaf and no leaves of $\lambda$ are isolated we can apply "Reeb Stability". That is, $\ell$ is approached by leaves of $\lambda$ that intersect each of the $P_{i}$. Say $P_{i}$ corresponds to $\sigma_{i} \subset T(\lambda)$. Then there exists a common initial segment $\tau \subset \sigma_{i}$. Let $\tau_{i}=\left(\sigma_{i}-\tau\right) \cup \tau^{+}$and consider the cyclic ordering on $\left\{-\tau, \tau_{2}, \ldots, \tau_{k}\right\}$ induced by the (strong) cyclic ordering on $T(\lambda)$. This is preserved by $f$ thus the linear order on $P_{2}, \ldots, P_{k}$ is also preserved by $f \mid X_{n}$. Now choose a product neighborhood $f\left(P_{1}\right)$ that connects $f\left(C_{0}\right)$ and $f\left(C_{1}\right)$ that is disjoint from $f\left(P_{i}\right)$ for $i \geq 2$. This extends $X_{n+1}$ in such a way the cyclic orderings induced from $T(\lambda)$ and $T\left(\lambda^{\prime}\right)$ are preserved.

3) Let $X_{n+1}=X_{n} \cup P \cup C$ with $P, C, Q_{i}$ as described in case 3 of Theorem 6.4. Since $C$ intersects each $Q_{i}$, the complementary region (which we optimistically denote $f(C)$ ) given by Proposition 6.1 intersects each $f\left(Q_{i}\right)$. Since the homeomorphism from $C$ to $f(C)$ must extend the map already defined on $\left(\cup Q_{i}\right) \cap C$ 
we need to know that the linear order on the $Q_{i}$ is preserved by $f \mid X_{n}$. This is established exactly as in Case 2 .

By Lemma $5.4 f$ can be extended over $P$.

The next lemma is the key step needed to show that the injective map produced in Proposition 6.7 can be chosen to be surjective.

Lemma 6.8. Let $\lambda, \lambda^{\prime}$ and $f:\left(\mathbb{R}^{2}, \lambda\right) \rightarrow\left(\mathbb{R}^{2}, \lambda^{\prime}\right)$ satisfy the hypothesis and conclusion of Proposition 6.7. Let $D$ and $E$ be disks in $\mathbb{R}^{2}$ such that $\partial D$ and $\partial E$ are transverse to $\lambda^{\prime}, D \subset f\left(\mathbb{R}^{2}\right)$, and $D$ is contained in the interior of $E$. Then there exists an isotopy $h_{t}:\left(\mathbb{R}^{2}, \lambda^{\prime}\right) \rightarrow\left(\mathbb{R}^{2}, \lambda^{\prime}\right)$ such that $h_{t}$ is the identity on $D$ for all $t$ and $h_{1}\left(f\left(\mathbb{R}^{2}\right)\right) \supset E$.

Proof. Choose a fibred neighborhood $N\left(\lambda^{\prime}\right)$ such that the components of $N\left(\lambda^{\prime}\right) \cap$ $E$ and $N\left(\lambda^{\prime}\right) \cap D$ are product laminations. Let $\left\{V_{j}\right\}$ be the components of $N\left(\lambda^{\prime}\right) \cap$ $(E-\operatorname{int}(D))$ which intersect both $\partial E$ and $\partial D$, and let $\left\{W_{k}\right\}$ the remaining components of $N\left(\lambda^{\prime}\right) \cap(E-\operatorname{int}(D))$. Let $V=\cup_{j} V_{j}$, and $W=\cup_{k} W_{k}$.

Since $f\left(\mathbb{R}^{2}\right)$ is an open set containing $D$ and all closed complementary regions of $\lambda^{\prime},\left(\mathbb{R}^{2}-f\left(\mathbb{R}^{2}\right)\right) \cap E \subset V \cup W$. Since $f\left(\mathbb{R}^{2}\right) \supset D$ it is straight forward to construct an isotopy $h_{t}$ fixing $D$ such that $h_{1}\left(f\left(\mathbb{R}^{2}\right)\right) \supset V$. Let $w_{k}$ be an $I$-bundle fibre of $W_{k}$. If $w_{k} \subset f\left(\mathbb{R}^{2}\right)$ it is easy to find an isotopy $h$ so that $h_{1}\left(f\left(\mathbb{R}^{2}\right)\right) \supset W_{k}$. All such isotopies can be performed simultaneously by an isotopy $h_{t}$. Let $Y$ be the union of those $W_{k}$ contained in $h_{1}\left(f\left(\mathbb{R}^{2}\right)\right)$. Further isotopies are required to fix $D \cup V \cup Y$.

Notationally we suppress the isotopies that have been done so far and suppose $w_{i} \not \subset f\left(\mathbb{R}^{2}\right)$. Since $\partial w_{i} \subset f\left(\mathbb{R}^{2}\right)$, and $w_{i}$ corresponds to an element of $T\left(\lambda^{\prime}\right)$, and $f_{*}$ is an isomorphism of order trees, there exists an efficient arc $\alpha$ of $\lambda^{\prime}$ such that $\partial \alpha=\partial w_{i}$ and $\alpha \subset f\left(\mathbb{R}^{2}\right)$. For simplicity, assume $\alpha \cap w_{i}=\partial \alpha$. In general it is enough to assume $\lambda \pitchfork w_{i}$ and then perform the following construction on subsegments of $\alpha$ and $w_{i}$.

By Lemma 5.4, $w_{i} \cup \alpha$ is the boundary of a disk $F \subset \mathbb{R}^{2}$ such that $\lambda^{\prime} \cap F$ is a product lamination of $F$. Let $X=F-f\left(\mathbb{R}^{2}\right)$. $X$ is a compact set that is a union of connected subsets of non-boundary leaves of $\lambda^{\prime}$ each of which intersect $w_{i}$. On the other hand, $D \cup V \cup Y$, the set which must remain fixed by the rest of the isotopy, does not intersect either $w_{i}$ or $X$. Thus it is possible to pick an efficient arc $\beta \subset F$ with $\partial \beta=\partial \alpha$ and $\beta \cap(X \cup D \cup V \cup Y)=\emptyset$.

The desired isotopy is one which maps a regular neighborhood of $\beta$ onto all of $W_{k}$.

Theorem 6.9. If two essential laminations $\lambda, \lambda^{\prime}$ of $\mathbb{R}^{2}$ have isomorphic cyclically ordered $\mathbb{R}$-order trees then, up to splitting along leaves, they are isomorphic.

Proof. It need only be shown that the injective map $f:\left(\mathbb{R}^{2}, \lambda\right) \rightarrow\left(\mathbb{R}^{2}, \lambda^{\prime}\right)$ given by Proposition 6.7 can be isotoped to a surjection.

Let $D_{1} \subset D_{2} \subset \ldots$ be disks whose union is $\mathbb{R}^{2}$ such that $D_{1} \subset f\left(\mathbb{R}^{2}\right)$, $D_{i} \subset \operatorname{int}\left(D_{i+1}\right)$ and $\partial D_{i} \pitchfork \lambda^{\prime}$ for all $i$. Inductively use Lemma 6.8 to choose isotopies $h^{i}$ that fix $D_{i}$ such that $h_{t}^{i}:\left(\mathbb{R}^{2}, \lambda^{\prime}\right) \rightarrow\left(\mathbb{R}^{2}, \lambda^{\prime}\right), h_{1}^{1}\left(f\left(\mathbb{R}^{2}\right)\right) \supset D_{2}$ and 
$h_{1}^{i}\left(h_{1}^{i-1}\left(\ldots h_{1}^{1} f\left(\mathbb{R}^{2}\right) \ldots\right) \supset D_{i+1}\right.$. The limit as $i$ tend to $\infty$ of $h_{1}^{i} h_{1}^{i-1} \ldots h_{1}^{1} f$ gives the desired homeomorphism.

\section{$\S 7$ Foliations of $\mathbb{R}^{2}$}

Theorem 6.9 generalizes, and is of course motivated by, the classical results of Haefliger and Reeb [HR]. The next proposition is used to explore the relationship between the objects which classify laminations, namely order trees, and the (possibly non-Hausdorff) 1-manifolds which were used in [HR] to classify foliations of the plane.

Proposition 7.1. If $\lambda$ is an essential lamination of $\mathbb{R}^{2}$, there is a foliation $\varphi$ of $\mathbb{R}^{2}$ such that each leaf of $\lambda$ is a leaf of $\varphi$.

Proof. Let $X_{1} \subset X_{2} \subset \ldots$ be the exhaustion of $\mathbb{R}^{2}$ given by Theorem 6.4 applied to $\lambda$. $\lambda$ will be inductively extended to a foliation.

If $C$ is a closed complementary region of $\lambda$ then $C$ is a $D^{2}$ missing boundary manifold, that is, $C$ is homeomorphic to a set $M$ with $\operatorname{int}(D) \subset M \subset D^{2}$. Since $\lambda$ is essential $M \neq D^{2}$.

It is easy to see that $\lambda \mid C$, that is $\partial C$, extends to a foliation $\varphi$ of $C$. In particular, let $\varphi \mid X_{1}$ be an extension of $\lambda \mid X_{1}$. Of course if $P$ is a product neighborhood of an efficient $\operatorname{arc} \lambda \mid P$ extends to a product foliation of $P$. Suppose that $\varphi$ has been defined on $X_{n}$ and consider the cases in Theorem 6.4.

1) If $X_{n+1}$ is obtained by thickening a product neighborhood $P, \varphi \mid P$ can be extended to the thickening.

2) If $X_{n+1}=X_{n} \cup P \cup C$ pick arbitrary extensions of $\lambda \mid P$ and $\lambda \mid C$ to give a foliation of $X_{n+1}$.

3) If $X_{n+1}=X_{n} \cup P \cup C$ let $Q_{1}, \ldots, Q_{k}$ and $C_{i}$ be as described in Case 3 of Theorem 6.4. For each $i$ let $Q_{i}^{\prime}$ be the portion of $Q_{i}$ between $C_{0}$ and $C_{i}$. $\varphi \mid Q_{1}^{\prime} \cup \cdots \cup Q_{i}^{\prime}$ extends to a foliation of $P$ by Lemma 5.4. Since $\lambda$ is essential, $C$ has exactly two boundary leaves which intersect $P$ and $\varphi \mid X_{n} \cup P$ may be extended to a foliation of each component of $C-P$.

Corollary 7.2. A cyclically ordered $\mathbb{R}$ order tree $T$ is a quotient of a (possibly non-Hausdorff) 1-manifold.

Proof. $T$ is realized by an essential lamination $\lambda$ of $\mathbb{R}^{2}$ which extends to a foliation $\varphi$ of $\mathbb{R}^{2}$. Hence $T \cong T(\lambda)$ is a quotient of the 1 -manifold $T(\varphi)$.

\section{$\S 8$ Essential laminations of $\mathbb{R}^{3}$}

The theory of codimension- 1 laminations of $\mathbb{R}^{3}$ is almost exactly the same as the theory in $\mathbb{R}^{2}$. The differences are:

1) In the definition of an essential lamination the leaves must be assumed to be planes.

2) The closed complementary regions must be assumed to be $B^{3}$ missing boundary manifolds, that is, they must be homeomorphic to a set $M$ such that $\operatorname{int}\left(B^{3}\right) \subset M \subset B^{3}$. 
3) The definition of the order tree associated to an essential lamination of $\mathbb{R}^{3}$ is unchanged, but there is no canonical cyclic ordering on the associated order tree.

By [GO, Theorem 6.1] it is not necessary to assume the ambient manifold is $\mathbb{R}^{3}$, for any simply connected manifold containing an essential lamination must be homeomorphic to $\mathbb{R}^{3}$.

The classification of these essential laminations is given by

Theorem 8.1. There is a 1-1 correspondence between homeomorphism classes of essential laminations of $\mathbb{R}^{3}$ and isomorphism classes of $\mathbb{R}$-order trees.

Proof. The proof in this case is essentially the same as the proof in dimension 2 , so we make only one remark on the "cyclically ordered" structure which is not needed in this dimension. Two non-compact $B^{3}$ missing boundary manifolds with the same (possibly infinite) number of boundary components, each of which is homeomorphic to $\mathbb{R}^{2}$, are homeomorphic. Moreover, any permutation of their boundary components may be realized by a homeomorphism. Thus it is possible to show that two essential laminations are equivalent without assuming isomorphic cyclic orderings on the associated order trees.

Essential laminations of $\mathbb{R}^{2}$ and $\mathbb{R}^{3}$ are related by the following result of [GK, Theorem 4.6]. The original version for foliations was proved by Palmeira $[\mathrm{P}]$.

Theorem 8.2. If $\Lambda$ is an essential lamination of $\mathbb{R}^{3}$ then there exists an essential lamination $\lambda$ of $\mathbb{R}^{2}$ such that $\left(\mathbb{R}^{3}, \Lambda\right) \cong\left(\mathbb{R}^{2}, \lambda\right) \times \mathbb{R}$.

Combining the last two theorems we see that there is a correspondence between expressing $\left(\mathbb{R}^{3}, \Lambda\right)$ as a product and choosing a cyclic ordering on $T(\Lambda)$. There may be an uncountable number of cyclic orderings on an $\mathbb{R}$-order tree.

\section{References}

[GK] D. Gabai and W. H. Kazez, Homotopy, isotopy and genuine laminations of 3-manifolds, Geometric Topology (William H. Kazez, ed.), vol. 1, Amer. Math. Soc./Internat. Press, 1997, pp. 123-138.

[GO] D. Gabai and U. Oertel, Essential laminations in 3-manifolds, Ann. of Math. (2) 130 (1989), 41-73.

[HR] A. Haefliger et G. Reeb, Variétés (non séparées) à une dimension et structures feuilletées du plan, Enseignement Math. (2) 3 (1957), 104-125.

[MS] J. W. Morgan and P. B. Shalen, Valuations, trees, and degenerations of hyperbolic structures, I., Ann. of Math. 120 (1984), 401-176.

[Pa] C. F. B. Palmeira, Open manifolds foliated by planes, Ann. of Math. 107 (1978), 109131.

253-37, California Institute of Technology, Pasadena, CA 91125

E-mail address: gabai@math.caltech.edu

University of Georgia, Athens, GA 30602

E-mail address: will@math.uga.edu 